OPEN ACCESS

Edited by:

Teng Ma,

Capital Medical University, China

Reviewed by:

Sunny Sharma,

The State University of New Jersey,

United States

Eva Bartova

Academy of Sciences of the Czech

Republic, Czechia

*Correspondence:

Shu-Yang Zhang

shuyangzhang103@nrdrs.org

Specialty section:

This article was submitted to Protein and RNA Networks,

a section of the journal

Frontiers in Molecular Biosciences

Received: 20 February 2021 Accepted: 14 July 2021

Published: 28 July 2021

Citation:

Han Y-C, Xie H-Z, Lu B, Xiang R-L,

Zhang $H-P, L i J-Y$ and Zhang S-Y (2021) Lipopolysaccharide Alters the

m6A Epitranscriptomic Tagging of

RNAs in Cardiac Tissue.

Front. Mol. Biosci. 8:670160.

doi: 10.3389/fmolb.2021.670160

\section{Lipopolysaccharide Alters the m6A Epitranscriptomic Tagging of RNAs in Cardiac Tissue}

\author{
Ye-Chen Han ${ }^{1}$, Hong-Zhi Xie ${ }^{1}$, Bo Lu ${ }^{1}$, Ruo-Lan Xiang ${ }^{2}$, Hai-Peng Zhang ${ }^{3}$, Jing-Yi Li ${ }^{1}$ and \\ Shu-Yang Zhang ${ }^{1 *}$ \\ ${ }^{1}$ Department of Cardiology, Peking Union Medical College Hospital, Chinese Academy of Medical Sciences and Peking Union \\ Medical College, Beijing, China, ${ }^{2}$ Department of Physiology and Pathophysiology, Peking University School of Basic Medical \\ Sciences, Beijing, China, ${ }^{3}$ Peking University Fifth School of Clinical Medicine (Beijing Hospital), Beijing, China
}

N6-methyladenosine $\left(\mathrm{m}^{6} \mathrm{~A}\right)$ modification plays important roles in the pathology of a variety of diseases. However, the roles of $\mathrm{m}^{6} \mathrm{~A}$ modification in sepsis-induced myocardial dysfunction are not well defined. Rats were divided into control and lipopolysaccharide (LPS)-induced sepsis group. Global $\mathrm{m}^{6} \mathrm{~A}$ levels of left ventricle tissue were measured by LC-MS/MS, and transcriptome-wide $\mathrm{m}^{6} \mathrm{~A}$ modifications were profiled using epitranscriptomic microarrays (mRNAs and IncRNAs). Bioinformatics analysis was conducted to understand the functional implications of $\mathrm{m}^{6} \mathrm{~A}$ modifications during sepsis. Methylated IncRNAs and mRNAs were measured by $m^{6} A$ single-base site qPCR. The global $\mathrm{m}^{6} \mathrm{~A}$ levels in left ventricle tissue were significantly decreased in the LPS group. While 27 transcripts (23 mRNAs and four IncRNAs) were hypermethylated, 46 transcripts (39 mRNAs and 7 IncRNAs) were hypomethylated in the LPS group. The mRNA expression of writers and readers was significantly decreased in the LPS group. The $\mathrm{m}^{6} \mathrm{~A}$ modification of Clec1b, Stk38I and Tnfrsf26 was associated with platelet activation and apoptotic pathways. Moreover, the decrease in $\mathrm{m}^{6} \mathrm{~A}$ modification of IncRNA XR_346,771 may be related to cation import in cardiac tissue. Our data provide novel information regarding changes to $\mathrm{m}^{6} \mathrm{~A}$ modifications in cardiac tissue during sepsis, and $\mathrm{m}^{6} \mathrm{~A}$ modifications might be promising therapeutic targets.

Keywords: N6-methyladenosine, sepsis, myocardial dysfunction, epitranscriptomics, IncRNA

\begin{abstract}
Abbreviations: BP, biological processes; CC, cellular components; ceRNA, Competing endogenous RNA; ELF3, epitheliumspecific ETS (ESE) transcription factors; FTO, Fat mass and obesity-associated protein; GO, Gene Ontology; HAKAI, E3 ubiquitin-protein ligase Hakai; HCC, epatocellular carcinoma; hnRNPA2B1, heterogeneous nuclear ribonucleoprotein A2B1; IGF2BP1/2/3, insulin-like growth factor 2 mRNA-binding proteins 1/2/3; KEGG, Kyoto Encyclopedia of Genes and Genomes; LC-MS/MS, Liquid chromatography-tandem mass spectrometry; IncRNAs, long non-coding RNAs; LPS, lipopolysaccharide; m6A, N6-methyladenosine; METTL14, methyltransferase-like 14; METTL3, methyltransferase like 3; MF, molecular functions; PPI, Protein-protein interaction; RBM15/15B, RNA binding motifs protein 15/15B; WTAP, Wilms tumor 1-associated protein; YTHDC1/2, YTH domain-containing reader proteins 1/2; YTHDF 2, YTH domain family 2; ZC3H13, zinc finger CCCH-type containing 13
\end{abstract}




\section{INTRODUCTION}

Sepsis is a life-threatening condition involving organ dysfunction that is caused by a dysregulated host response to infection (Cohen et al., 2015), It has been reported that approximately $50 \%$ of patients with septic shock are diagnosed with septic cardiomyopathy, which causes myocardial dysfunction, and these patients tend to have poor prognoses (Zaky et al., 2014). Myocardial dysfunction is an important factor that contributes to the high mortality rate of sepsis. Therefore, it is necessary to elucidate the pathogenesis of myocardial dysfunction in order to develop new treatment strategies to reduce the mortality rate of sepsis.

To date, a series of reversible posttranscriptional modifications located in RNAs have gained increasing attention. More than 140 chemical modifications have been discovered in RNAs. The modifications range from simple methylation or isomerisation, such as N6-methyladenosine $\left(\mathrm{m}^{6} \mathrm{~A}\right)$, 5-methylcytosine (m5C), N1-methyladenosine $\left(\mathrm{m}^{1} \mathrm{~A}\right)$, pseudouridine $(\Psi), 5$-methyluridine $\left(\mathrm{m}^{5} \mathrm{U}\right), 1$-methylguanosine, and 7-methylguanosine $\left(\mathrm{m}^{1} \mathrm{G}\right.$ and $\mathrm{m}^{7} \mathrm{G}$, respectively) and inosine (I), to complex multistep chemical modifications, such as $\mathrm{N}^{6}$ threonylcarbamoyladenosine and 5-methoxycarbonyl-methyl2-thiouridine $\left(\mathrm{mcm} 5 \mathrm{~s}^{2} \mathrm{U}\right)$ ( $\left.\mathrm{Pan}, 2018\right)$. Among them, the $\mathrm{m}^{6} \mathrm{~A}$ is one of the most abundant and influential modifications in eukaryotes. $\mathrm{M}^{6} \mathrm{~A}$ modification is involved in regulating a variety of posttranscriptional events, including pre-miRNA processing and RNA stability, translation and alternative splicing (Chokkalla et al., 2019). $\mathrm{M}^{6} \mathrm{~A}$ modifications occur via the $\mathrm{m}^{6} \mathrm{~A}$ methyltransferases called "writers"; they are removed by the demethylases called "erasers" and are recognized by m6Abinding proteins called "readers". The orchestrated interplay among writers, erasers, and readers drives the dynamics and outcomes of the $\mathrm{m}^{6} \mathrm{~A}$ modification of RNAs. An increasing number of studies have reported that $\mathrm{m}^{6} \mathrm{~A}$ modification plays important roles in biological and pathophysiological processes, such as tumorigenesis, embryonic stem cell differentiation, and viral infection (Kennedy et al., 2016). In mouse GC-1 SPG cells, meclofenamic acid inhibits spermatogonial proliferation by affecting CDKs expression through a $\mathrm{m}^{6} \mathrm{~A}$-dependent mRNA degradation pathway (Huang et al., 2019). In liver cancer, overexpression of METTL3 leads to the degradation of suppressor of cytokine signalling two and promotes tumor growth (Lin et al., 2019).

$\mathrm{M}^{6} \mathrm{~A}$ modification is also associated with the pathophysiological processes of cardiac differentiation and a variety of cardiovascular diseases. Methyltransferase-like 3 (METTL3) is a major factor involved in abnormal $\mathrm{m}^{6} \mathrm{~A}$ modification. Silencing METTL3 enhances the autophagic flux and represses apoptosis in hypoxia/reoxygenation-treated cardiomyocytes (Song et al., 2019). Overexpression METTL3 increased $\mathrm{m}^{6} \mathrm{~A}$ levels in mRNAs isolated from transverse aortic constriction (TAC) mice hearts. TAC inducd pathological hypertrophic cellular growth was attenuated in hearts of METTL3-overexpressing mice, as evidenced by the crosssectional area of myocytes (Kmietczyk et al., 2019). In addition, aging caused METTL3/METTL14 down-regulation in aorta and atria in male animals while the differentiation-induced increased level of METTL16 (Arcidiacono et al., 2020). Prabhu M et al. found that downregulation of fat mass and obesity associated protein (FTO) expression in failing mammalian hearts and hypoxic cardiomyocytes increased the $\mathrm{m}^{6} \mathrm{~A}$ modification of RNAs and reduced the contractile function of cardiomyocytes (Mathiyalagan et al., 2019). FTO also played a vital role in cardiac contractile function during homeostasis and remodeling. FTO overexpression attenuated the ischemiainduced elevation in $\mathrm{m}^{6} \mathrm{~A}$ modification and significantly improved cardiac function of post-myocardial infraction (Mathiyalagan et al., 2019). Berulava et al. reported that differently expressed genes of $\mathrm{m}^{6} \mathrm{~A}$ methylation are involved in heart failure development. Bioinformatics analysis has revealed that the differentially $\mathrm{m}^{6} \mathrm{~A}$ are mainly involved in metabolism and cardiac signaling (Berulava et al., 2020). However, the regulatory role of the $\mathrm{m}^{6} \mathrm{~A}$ modification in cardiac function is still unclear.

The contraction of the ventricle transports blood to the capillaries of the body or lungs, while the contraction of the atria send blood into the ventricle. In addition, the right ventricle mainly supplies blood to the lungs, and the blood in the right ventricle is venous blood. The left ventricle mainly supplies blood to the organs and tissues of the body, and the blood in the left ventricle is arterial blood. Therefore, the function of the left ventricle is very important to maintain organ perfusion. If the left ventricular function declines, it will leads to organ perfusion insufficiency and, eventually, organ failure. In this direction, we focused on the mechanism underlying left ventricle injury in sepsis to find any potential clinical significance for improving organ perfusion. The overall goal of this study was to explore a new layer of epigenetic alterations through the genome-wide screening of the altered $\mathrm{m}^{6} \mathrm{~A}$-tagged transcript profiles in lipopolysaccharide (LPS)-induced myocardial dysfunction. We successfully mapped $\mathrm{m}^{6} \mathrm{~A}$ transcripts in left ventricle tissue in LPS-induced sepsis. Some potential roles of the $\mathrm{m}^{6} \mathrm{~A}$ modification in the physiological and pathological mechanisms of sepsis were revealed.

\section{MATERIALS AND METHODS}

\section{Animals}

Eight-week-old male Wistar rats weighing 250-350 g were used in this study. All the rats used in our study were obtained from Charles River Laboratories (Beijing, China). Water and standard laboratory food were freely available to the animals. The Experimental Animal Welfare Ethics Branch and the Biomedical Ethics Committee of Peking University approved our study protocol (LA 2020343). All the procedures for handling the animals were in accordance with the Guide for the Care and Use of Laboratory Animals (NIH Publication No. 85-23, revised 1996).

After acclimating for 1 week, $10 \mathrm{Wistar}$ rats were randomly divided into two groups. The rats in the LPS group $(n=5)$ were intraperitoneally injected with LPS $(10 \mathrm{mg} / \mathrm{kg}, 5 \mathrm{mg}$ LPS dissolved in $1 \mathrm{ml} 0.9 \%$ saline). The rats in the control group $(n=5)$ were intraperitoneally injected with $0.9 \%$ saline $(2 \mathrm{ml} / \mathrm{kg})$. 


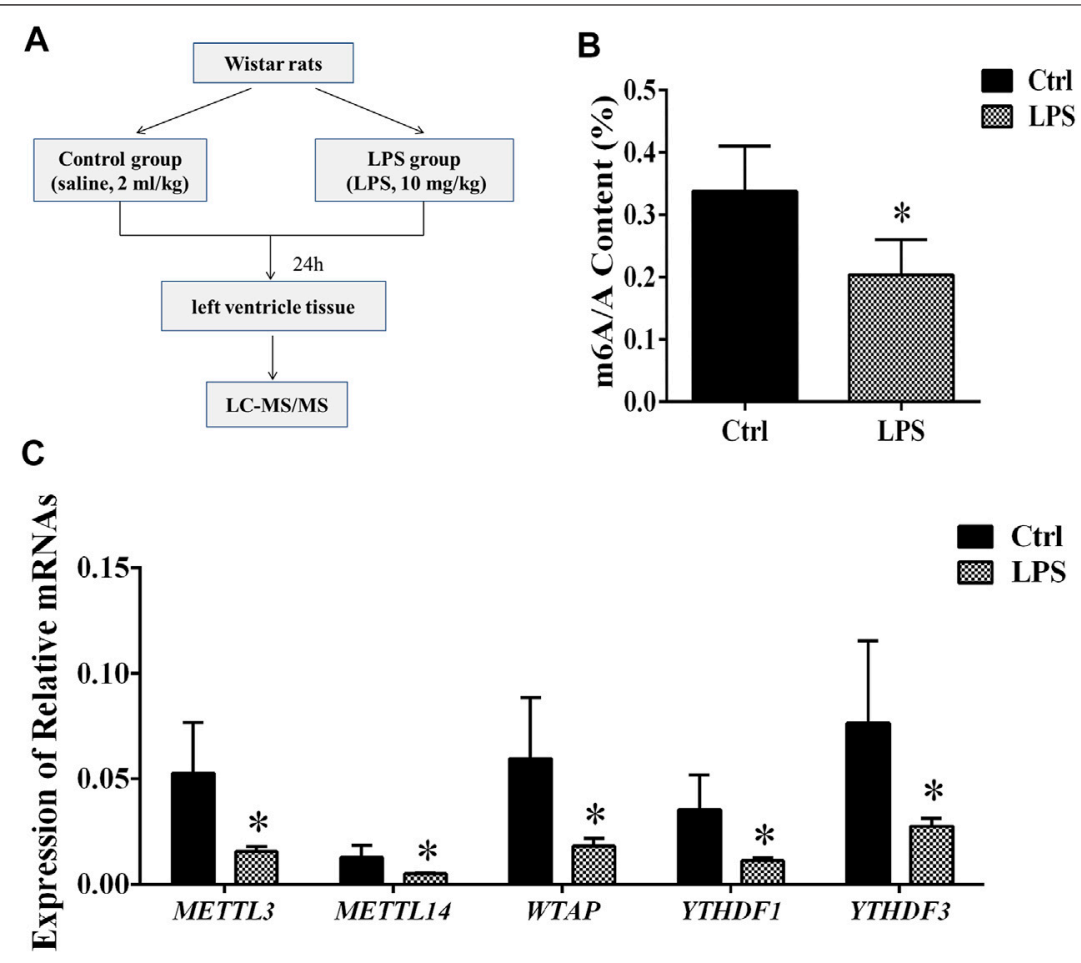

FIGURE 1 | Global level of $m^{6} A$ modification and mRNA levels of enzymes in left ventricle tissue. (A) Flowchart of tissue collection. (B) Global level of $m^{6} A$ modification measured by LC-MS/MS in left ventricle tissue. (C) Relative expression mRNA levels of m ${ }^{6} \mathrm{~A}$-related enzymes in left ventricle tissue during sepsis. The mRNA levels were determined by qRT-PCR and normalized by GAPDH. * $p<0.05$ versus the Ctrl group. Ctrl, control; LPS, lipopolysaccharide.

24 hours after LPS injection, mean blood pressure was measured by the tail-cuff method with a noninvasive blood pressure measurement system. The left ventricle tissue samples were immediately transferred to liquid nitrogen and stored at $-80^{\circ} \mathrm{C}$ for preservation.

\section{RNA Extraction and Quality Control}

Total RNA was isolated from the left ventricle tissues of the LPS $(n=5)$ and control $(n=5)$ groups using TRIzol Reagent (Invitrogen, United States). The quantity and purity of the total RNA samples were measured by a NanoDrop ND-1000 (ThermoFisher, United States).

\section{Quantification of Global $\mathbf{m}^{6} \mathrm{~A}$ Levels}

Liquid chromatography-tandem mass spectrometry (LC-MS/ MS)-based mRNA modification detection was performed according to the Aksomics standard protocol. Briefly, $5 \mu \mathrm{g}$ of total RNA from the heart tissues of the LPS $(n=5)$ and control $(n=5)$ groups was used to isolate mRNA using the NEBNext Poly(A) mRNA Magnetic Isolation Module (NEB, E7490, United States). The purified mRNA was quantified using the Qubit RNA HS Assay kit (ThermoFisher, United States) and digested to single dephosphorylated nucleosides by an enzyme mixture. Pretreated nucleosides solution was deproteinized using Satorius 10,000-Da MWCO spin filter. LC-MS/MS analysis was performed on an Agilent 6460 QQQ mass spectrometer with an Agilent 1260 HPLC system using Multi reaction monitoring
(MRM) detection mode (Agilent, United States. The nucleosides were quantified by using retention time and the nucleoside to base ion mass transitions of 268-136 (A) and 282-150 ( $\left.\mathrm{m}^{6} \mathrm{~A}\right)$. Quantification was performed in comparison with the standard curve obtained from pure nucleoside standards running with the same batch of samples. The $\mathrm{m}^{6} \mathrm{~A}$ level was calculated as the ratio of $\mathrm{m}^{6} \mathrm{~A}$ to $\mathrm{A}$ based on the calibrated concentrations.

\section{Quantitative Real-Time PCR}

After extraction, the total RNA was reverse-transcribed into cDNA using SuperScriptTM III Reverse Transcriptase (Invitrogen) according to the manufacturer's instructions. Amplification and detection were performed using $2 \times$ SYBR Green PCR Master Mix (Arraysta, United States) on a ViiA 7 Real-Time PCR System (Applied Biosystems). The sequences of the gene-specific primers are listed in Supplementary Table S1. GAPDH was used as the control housekeeping gene.

\section{Western Blot Analysis}

Protein extraction of left ventricle tissue and the protein concentration was determined by Bradford method (M\&C Gene Technology Ltd.). The protein samples were then subjected to SDS-polyacrylamide gel electrophoresis (SDSPAGE) and transferred to polyvinylidene difluoride membrane. The membranes were probed with antibodies of interest. The antibodies used were as follows: METTL3, 

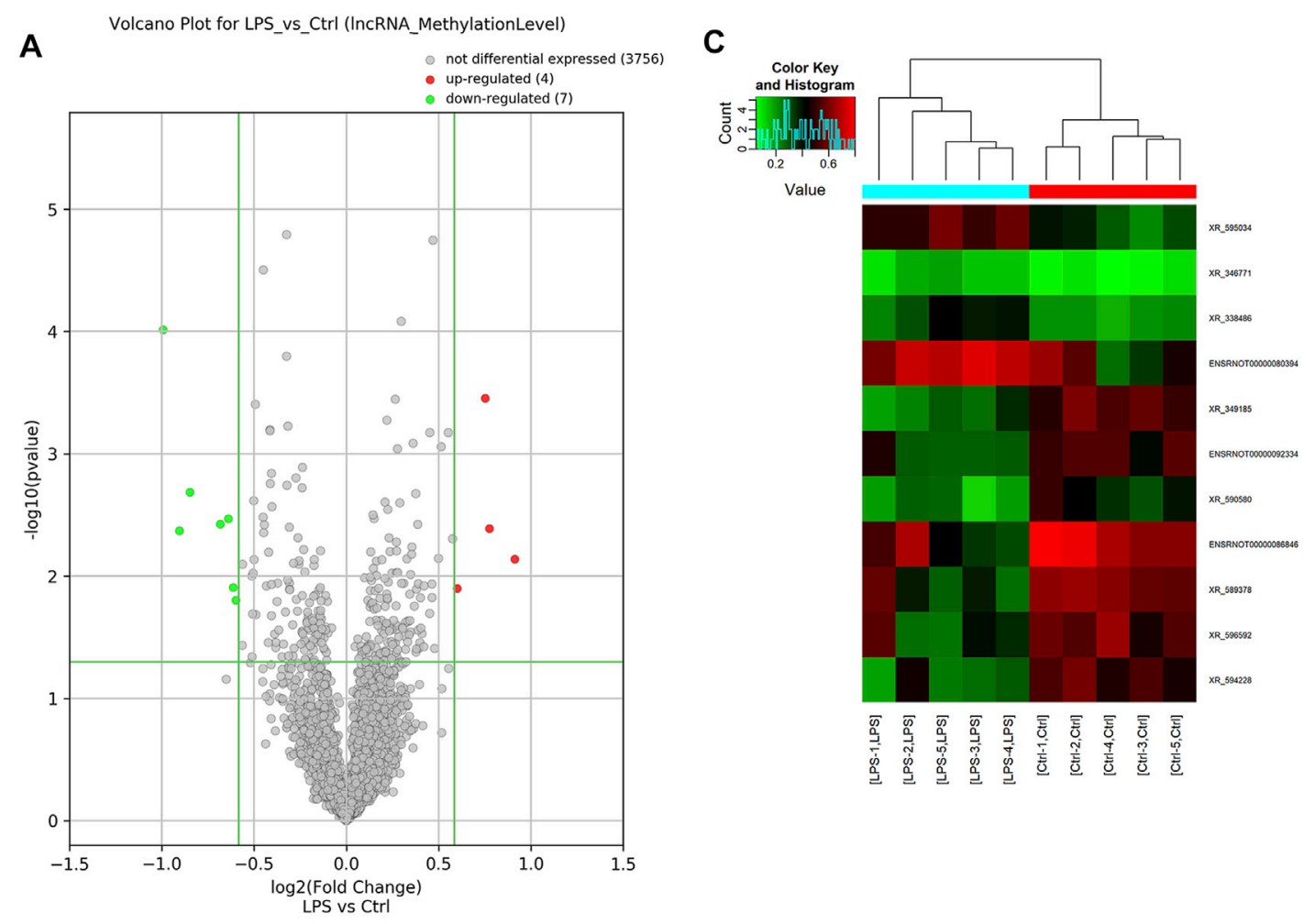

B

Volcano Plot for LPS_vs_Ctrl (mRNA_MethylationLevel)
not differential expressed (19168)
- up-regulated (23)

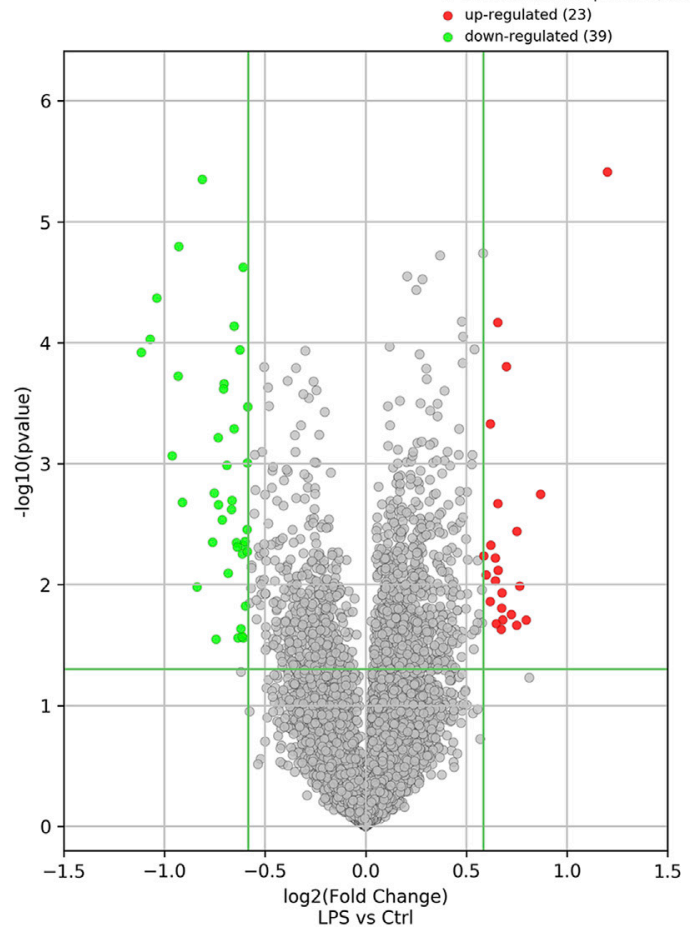

D
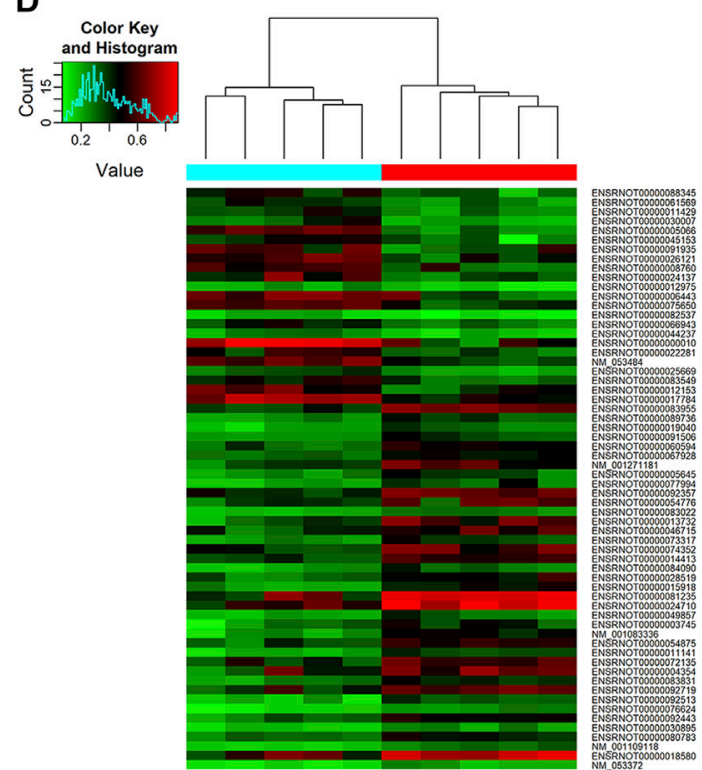

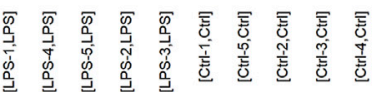

PS vs Ctr

FIGURE 2 | M ${ }^{6}$ A modification profiles of IncRNA\& mRNA in LPS and control group. (A) Volcano plots showing the IncRNAs that were differentially methylated between LPS and control group with statistical significance. (B) Volcano plots showing the mRNAs that were differentially methylated between LPS and control group with statistical significance (fold changes $\geq 1.5$ and $p<0.05$ ). (C) Hierarchical clustering analysis the differentially methylated IncRNAs. (D) Hierarchical clustering analysis the differentially methylated mRNAs. Ctrl, control; LPS, lipopolysaccharide. 
TABLE 1 | The detailed information of the hyper-methylated and hypo-methylated IncRNAs.

\begin{tabular}{|c|c|c|c|c|c|c|c|}
\hline Transcript_ID & Type & Gene symbol & RNA length & Locus & Regulation & Fold change & $p$-value \\
\hline XR_346771 & IncRNA & LOC102552786 & 1764 & chr5:148464894-148467759:- & Hyper & 1.882651632 & 0.007280323 \\
\hline XR_338486 & IncRNA & LOC102547952 & 997 & chr10:14824098-14825718:+ & Hyper & 1.711575396 & 0.004096892 \\
\hline XR_595034 & IncRNA & LOC103693543 & 1931 & chr15:32741179-32746755:- & Hyper & 1.684697484 & $0.000352,285$ \\
\hline ENSRNOT00000080394 & IncRNA & AABR07059875.4 & 185 & chr4:39636928-39637206:- & Hyper & 1.516656629 & 0.012635768 \\
\hline XR_349185 & IncRNA & LOC102548542 & 1841 & chr9:93297368-93301959:- & Hypo & 0.502693743 & 0.000096980 \\
\hline XR_590580 & IncRNA & LOC103691283 & 540 & chr12:22434074-22434701:- & Hypo & 0.534479482 & 0.004268491 \\
\hline XR_594228 & IncRNA & LOC103690476 & 702 & chr8:130823601-130827254:+ & Hypo & 0.555894587 & $0.00206,689$ \\
\hline XR_589378 & IncRNA & LOC103690602 & 719 & chr11:79090944-79093774:+ & Hypo & 0.623167691 & $0.00376,447$ \\
\hline ENSRNOT00000092334 & IncRNA & Comp & 991 & chr16:20803585-20805482:- & Hypo & 0.642164861 & 0.003406754 \\
\hline ENSRNOT00000086846 & IncRNA & AABR07026021.1 & 524 & chr16:59517000-59524544:+ & Нyро & 0.654135708 & 0.012402534 \\
\hline XR_596592 & IncRNA & LOC102548402 & 5498 & chr17:5486419-5494314:- & Нyро & 0.660712559 & 0.015797815 \\
\hline
\end{tabular}

TABLE 2 | The detailed information of the top ten hyper-methylated and top ten hypo-methylated mRNAs.

Transcript_ID

ENSRNOT00000005066

ENSRNOTO00000000010

ENSRNOT00000030007

ENSRNOT00000006443

ENSRNOTO0000008760

ENSRNOT00000045153

ENSRNOT00000082537

NM_053484

ENSRNOTO00000024137

ENSRNOT00000088345

NM 001083336

ENSRNOT00000076624

ENSRNOTO0000015918

ENSRNOT00000092513

ENSRNOT00000011141

NM_001109118

ENSRNOTO0000013732

ENSRNOTO0000077994

ENSRNOT00000083955

NM_053372
Type

protein_coding

protein_coding

protein_coding

protein_coding

protein_coding

protein_coding

protein_coding

protein_coding

protein_coding

protein_coding

protein coding

protein_coding

protein_coding

protein_coding

protein_coding

protein_coding

protein_coding

protein_coding

protein_coding

protein_coding
GeneSymbol

\section{Cfap52}

Tmco5b

Ptk2b

Rtn4

$\mathrm{Cd} 44$

LOC103689983

Clec1b

Gas7

Drd4

AABR07052508.1

Stk38!

Slpil3

Tnfrsf21

Rps15a

Gprc5d

Elov12

116

Ssr1

Fkbp11

Slpi

\begin{tabular}{c} 
RNA length \\
\hline 2237 \\
1361 \\
3848 \\
4665 \\
1437 \\
1223 \\
958 \\
6796 \\
1416 \\
258 \\
2406 \\
663 \\
4350 \\
528 \\
1402 \\
3731 \\
1045 \\
1196 \\
717 \\
667
\end{tabular}

667
Locus

chr10:54470835-54512169:-

chr3:104749051-104765436:+ chr15:42827310-42947656:chr14:114126966-114174458:+ chr3:92697833-92749121:chrX:158623240-158655198: chr4:163162211-163170466:+ chr10:54086843-54240798:+

chr1:214278296-214281483:+ chr3:58380143-58386375:+ chr4:181027212-181087530:+ chr3:160774855-160777092:chr9:20546159-20621051: chr1:187759865-187766670: chr4:168872897-168884886:chr17:21382461-21422410:+ chr4:3043231-3047807:+ chr17:27496353-27510918:+ chr7:140398253-140401686:chr3:160799979-160802228:-

Regulation
Hyper
Hyper
Hyper
Hyper
Hyper
Hyper
Hyper
Hyper
Hyper
Hyper
Hypo
Hypo
Hypo
Hypo
Hypo
Hypo
Hypo
Hypo
Hypo
Hypo

Fold change

$p$-value

$2.299100294 \quad 0.000003881$

$\begin{array}{ll}1.8263071 & 0.001794981\end{array}$

$1.738045027 \quad 0.019677042$

$\begin{array}{ll}1.699559099 & 0.010322126\end{array}$

$\begin{array}{ll}1.683312914 & 0.003639311\end{array}$

$\begin{array}{ll}1.68214218 & 0.021749985\end{array}$

$\begin{array}{ll}1.651427112 & 0.017707274\end{array}$

$\begin{array}{ll}1.623629355 & 0.000157904\end{array}$

$\begin{array}{ll}1.603101886 & 0.019629213\end{array}$

$\begin{array}{ll}1.599183502 & 0.011739780\end{array}$

$\begin{array}{ll}0.461936557 & 0.000120507\end{array}$

$\begin{array}{ll}0.476197276 & 0.000093915\end{array}$

$\begin{array}{ll}0.487217803 & 0.000042853\end{array}$

$\begin{array}{ll}0.513673869 & 0.000863271\end{array}$

$\begin{array}{ll}0.524397823 & 0.000189455\end{array}$

$\begin{array}{ll}0.525563958 & 0.000016059\end{array}$

$\begin{array}{ll}0.532128419 & 0.002095281\end{array}$

$0.559623758 \quad 0.010488981$

$\begin{array}{lll}0.569855508 & 0.000004472\end{array}$

$\begin{array}{ll}0.590419522 & 0.004477119\end{array}$
METTL14, Wilms tumor 1-associated protein (WTAP), and YTH N6-methyladenosine RNA binding protein 1 (YTHDF1), YTHDF3 (all1:1,000; Cell Signal Technology), and $\beta$-actin (1: 4,000; Abcam).

\section{$M^{6}$ A mRNA\&IncRNA Epitranscriptomic Microarray}

Sample preparation and microarray hybridization were performed based on Arraystar's standard protocols (Arraystar). Briefly, total RNA from the heart tissues of the LPS $(n=5)$ and control $(n=5)$ groups was immunoprecipitated with an anti- $\mathrm{m}^{6} \mathrm{~A}$ antibody (Synaptic Systems, 202003). The modified RNAs immunoprecipitated by the magnetic beads were labelled as "IP", and the unmodified RNAs in the supernatant were labelled as "Sup". The "IP" and "Sup" RNAs were labeled with Cy5 and Cy3, respectively, as cRNAs in separate reactions using the Arraystar Super RNA Labeling Kit (ArrayStar). The cRNAs were combined and hybridized onto an Arraystar Rat mRNA\&lncRNA Epitranscriptomic Microarray $(4 \times 44 \mathrm{~K}$,
Arraystar) that contained $27,770 \mathrm{mRNA}$ and 10,582 lncRNA degenerate probes. The hybridized arrays were scanned in twocolor channels by an Agilent Scanner G2505C.

\section{Microarray Data Analysis}

Agilent Feature Extraction software (version 11.0.1.1, United States) was used to analyze the acquired array images. Each probe signal was evaluated and flagged as present, absent or marginal in at least 5 out of 10 samples. The raw intensities of the IP (immunoprecipitated, Cy5-labeled) and Sup (supernatant, Cy3-labeled) samples were normalized by the average of $\log 2-$ scaled spike-in RNA intensities. The differentially $\mathrm{m}^{6} \mathrm{~A}$-methylated RNAs between the LPS and control groups were identified by filtering with thresholds of fold change $>1.5$ and statistical significance $(p<0.05)$.

\section{Bioinformatics Analysis}

Gene Ontology (GO) and Kyoto Encyclopedia of Genes and Genomes (KEGG) analyses were performed using some package in the $\mathrm{R}$ environment for statistical computing and graphics. The 


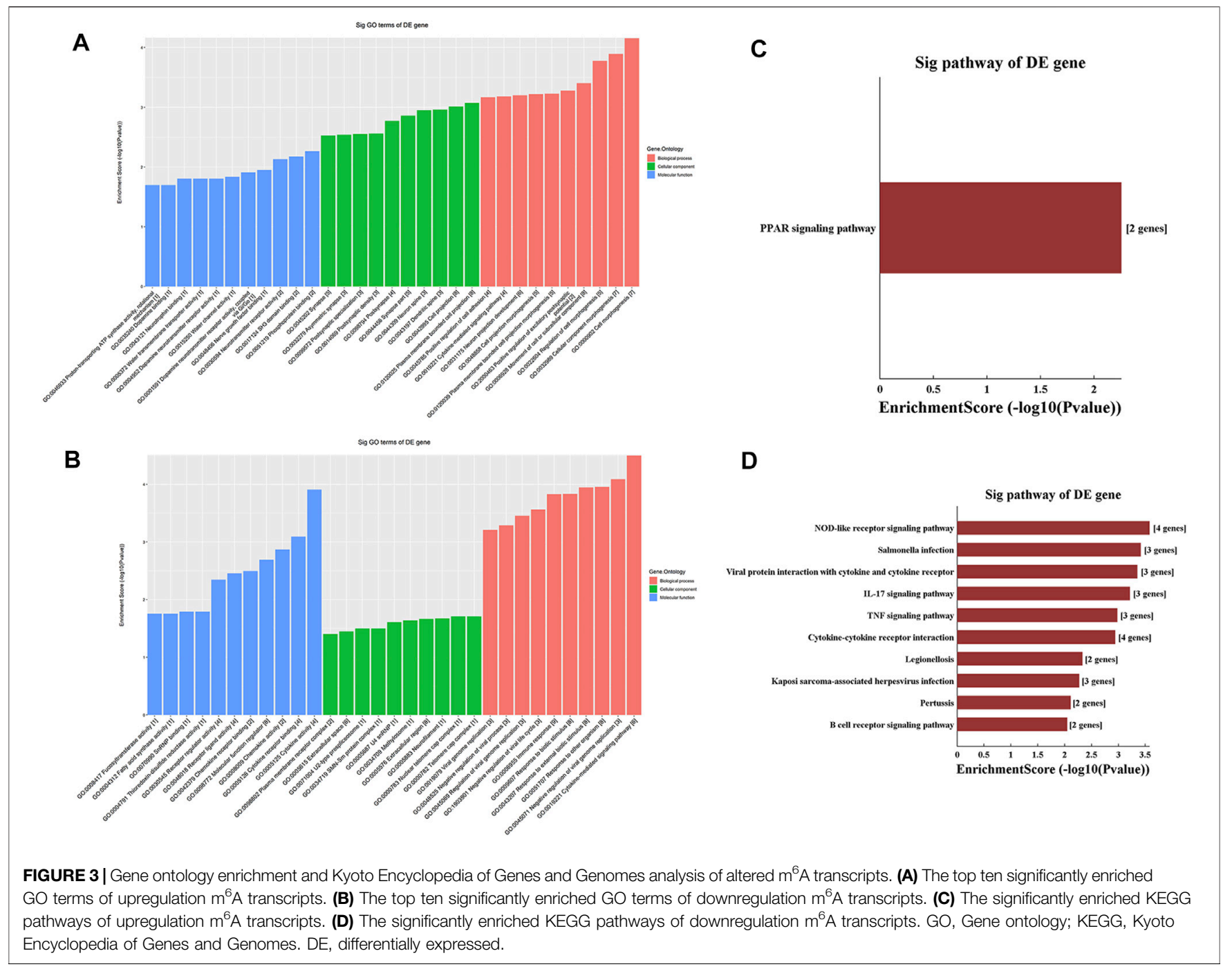

differentially $\mathrm{m}^{6} \mathrm{~A}$-methylated mRNAs, as well as the downstream mRNAs predicted by the competing endogenous RNA (ceRNA) network, were classified into different GO terms and enriched in certain biological pathways. Protein-protein interaction (PPI) analysis was performed using the STRING database (https:// string-db.org). The SRAMP database (http://www.cuilab.cn/ sramp) was used to predict the number of $\mathrm{m}^{6} \mathrm{~A}$ sites on the differentially $\mathrm{m}^{6} \mathrm{~A}$-methylated transcripts (Zhou et al., 2016).

\section{$M^{6}$ A Single-Base Site qPCR}

Before amplification and detection, the LPS $(n=5)$ and control $(n=5)$ group samples were treated with an Escherichia coli toxin and RNA endoribonuclease, MazF. MazF is reported to be sensitive to $\mathrm{m}^{6} \mathrm{~A}$ modification within the ACA motif (Imanishi et al., 2017). The MazF-digested mRNA samples and the nondigested samples were subjected to reverse transcription using SuperScriptTM III Reverse Transcriptase (Invitrogen) for qPCR as described above. The targeted lncRNAs and mRNAs were predicted by SRAMP to identify the ACA motif and $\mathrm{m}^{6} \mathrm{~A}$ position. The primer sequences specific for the methylated lncRNAs and mRNAs are listed in Supplementary Table S2. The relative expression levels were calculated using the $2^{-\triangle \triangle \mathrm{Ct}}$ method, and the tested genes were calibrated with MazF as follows:

$$
\% \text { MazF- }=\left(2^{\wedge}-C t_{M a z F+}\right) /\left(2^{\wedge}-C t_{M a z F-}\right) \times 100 \%
$$

\section{Competing Endogenous RNA Network Construction}

The targeted lncRNAs were verified by $\mathrm{m}^{6} \mathrm{~A}$ single-base site qPCR to construct the ceRNA network. Only three steps were used for ceRNA network construction. First, the potential target microRNAs of lncRNAs were predicted with Aksomics's homemade miRNA target prediction software based on TargetScan and miRanda (Enright et al., 2003; Pasquinelli, 2012). Second, the target genes of the miRNAs involved in the lncRNA-miRNA interaction network were predicted with the database described above. Finally, the lncRNA-microRNA- 
TABLE 3 | Gene Ontology analysis of the top ten hyper-methylated mRNAs.

\begin{tabular}{|c|c|c|c|c|c|c|}
\hline GO.ID & Term & Ontology & Count & $p$-value & Fdr & Enrichment score \\
\hline GO:0000902 & Cell morphogenesis & Biological process & 7 & 7.0344E-05 & 0.324473551 & 4.152772946 \\
\hline GO:0032989 & Cellular component morphogenesis & Biological process & 7 & 0.000128477 & 0.324473551 & 3.891174337 \\
\hline GO:0022604 & Regulation of cell morphogenesis & Biological process & 5 & 0.000166488 & 0.324473551 & 3.778618248 \\
\hline GO:0006928 & Movement of cell or subcellular component & Biological process & 8 & 0.000393 & 0.324473551 & 3.405607496 \\
\hline GO:2000463 & Positive regulation of excitatory postsynaptic potential & Biological process & 2 & 0.000525054 & 0.324473551 & 3.279795677 \\
\hline GO:0120,039 & Plasma membrane bounded cell projection morphogenesis & Biological process & 5 & 0.00058802 & 0.324473551 & 3.230607569 \\
\hline GO:0048858 & Cell projection morphogenesis & Biological process & 5 & 0.000599981 & 0.324473551 & 3.22186279 \\
\hline GO:0031175 & Neuron projection development & Biological process & 6 & 0.000627138 & 0.324473551 & 3.202636833 \\
\hline GO:0019221 & Cytokine-mediated signaling pathway & Biological process & 4 & 0.000657648 & 0.324473551 & 3.18200643 \\
\hline GO:0045785 & Positive regulation of cell adhesion & Biological process & 4 & 0.00067698 & 0.324473551 & 3.169424277 \\
\hline GO:0120,025 & Plasma membrane bounded cell projection & Cellular component & 8 & 0.000840205 & 0.232167614 & 3.075614779 \\
\hline GO:0042995 & Cell projection & Cellular component & 8 & 0.000972547 & 0.232167614 & 3.012089301 \\
\hline GO:0043197 & Dendritic spine & Cellular component & 3 & 0.001088661 & 0.232167614 & 2.963107415 \\
\hline GO:0044309 & Neuron spine & Cellular component & 3 & 0.001120031 & 0.232167614 & 2.950770139 \\
\hline GO:0044456 & Synapse part & Cellular component & 5 & 0.001379925 & 0.232167614 & 2.860144529 \\
\hline GO:0098794 & Postsynapse & Cellular component & 4 & 0.001689647 & 0.232167614 & 2.772204087 \\
\hline GO:0014069 & Postsynaptic density & Cellular component & 3 & 0.002740157 & 0.232167614 & 2.562224576 \\
\hline GO:0099572 & Postsynaptic specialization & Cellular component & 3 & 0.002796098 & 0.232167614 & 2.55344754 \\
\hline GO:0032279 & Asymmetric synapse & Cellular component & 3 & 0.002881334 & 0.232167614 & 2.540406329 \\
\hline GO:0045202 & Synapse & Cellular component & 5 & 0.002960384 & 0.232167614 & 2.528652018 \\
\hline GO:0051219 & Phosphoprotein binding & Molecular function & 2 & 0.005431961 & 1 & 2.265043371 \\
\hline GO:0017124 & SH3 domain binding & Molecular function & 2 & 0.006663867 & 1 & 2.176273674 \\
\hline GO:0030594 & Neurotransmitter receptor activity & Molecular function & 2 & 0.007384559 & 1 & 2.131675429 \\
\hline GO:0048406 & Nerve growth factor binding & Molecular function & 1 & 0.011176355 & 1 & 1.951699809 \\
\hline GO:0001591 & Dopamine neurotransmitter receptor activity, coupled via Gi/Go & Molecular function & 1 & 0.012287459 & 1 & 1.910537933 \\
\hline GO:0015250 & Water channel activity & Molecular function & 1 & 0.014506118 & 1 & 1.838448788 \\
\hline GO:0004952 & Dopamine neurotransmitter receptor activity & Molecular function & 1 & 0.015613677 & 1 & 1.806494817 \\
\hline GO:0005372 & Water transmembrane transporter activity & Molecular function & 1 & 0.015613677 & 1 & 1.806494817 \\
\hline GO:0043121 & Neurotrophin binding & Molecular function & 1 & 0.015613677 & 1 & 1.806494817 \\
\hline GO:0035240 & Dopamine binding & Molecular function & 1 & 0.02003213 & 1 & 1.698272881 \\
\hline
\end{tabular}

mRNA interaction networks were constructed by Cytoscape v2.8.3. The targeted mRNAs were also analyzed by GO and KEGG to completely understand the ceRNA effects.

\section{Statistical Analysis}

The statistical significance of the GO and KEGG analysis of mRNAs enrichment were calculated by Fisher's exact test $p<0.05$ and $-\log 10(p)$ transformed as the enrichment score as well as mRNAs predicted by the ceRNA network for GO and KEGG analysis. The significance of the differences in the expression and methylation levels between the LPS and control groups was evaluated with an unpaired two-sided $t$-test for LC-MS/MS, qRT-PCR, microarray analysis and $\mathrm{m}^{6} \mathrm{~A}$ single-base site $\mathrm{qPCR}$, and the recommended $p$-value threshold was less than 0.05 . "“” indicates a significant difference compared with control group $(p<0.05)$.

\section{RESULTS}

\section{The Global Levels of $\mathrm{m}^{6} \mathrm{~A}$ Modification Were Decreased}

In about $24 \mathrm{~h}$ after giving intraperitoneal injection of LPS, we measured the MAP of rats. As previous paper, the rats with a MAP declined to $25-30 \%$ or below were chosen as the sepsis model (Nie et al., 2020). Furthermore, signs of shock such as lassitude, tachycardia and a sharp drop in body temperature were observed in all sepsis rats. In the control group, there was no significant change in MAP in rats injected with saline. Previous study showed that there is a positive relationship between MAP and heart function (Yang et al., 2018; Zhang et al., 2019). Therefore, we isolated heart tissue and chose the left ventricle for identification of $\mathrm{m}^{6} \mathrm{~A}$ modification levels (Figure 1A). There was no measurement of cardiac function or sepsis physiology in these experiments.

To determine the difference in the $\mathrm{m}^{6} \mathrm{~A}$ modification levels between the LPS and control groups, we used LC-MS/MS to measure these levels. We found that the global $\mathrm{m}^{6} \mathrm{~A}$ modification level in the rat left ventricle tissues from the LPS group was significantly decreased compared with control group (Figure 1B).

\section{The Expression of $\mathrm{m}^{6} \mathrm{~A}$ Writers and Readers Was Downregulated}

The $\mathrm{m}^{6} \mathrm{~A}$ methylation modification is dynamically modulated by RNA methyltransferases, RNA-binding proteins and demethylases. We analyzed the mRNAs level of RNA methyltransferases (writers: METTL3, METTL14, and WTAP), RNA-binding proteins (readers: YTHDF 1 and YTHDF 3) and a demethylase (eraser: FTO). It was found that the expression of the METTL3/METTL14/WTAP/YTHDF 1, and YTHDF 3 in the LPS group was significantly downregulated compared with control group. However, the expression of FTO has no significant 
TABLE 4 | Gene Ontology analysis of the top ten hypo-methylated mRNAs.

\begin{tabular}{|c|c|c|c|c|c|c|}
\hline GO.ID & Term & Ontology & Count & $p$-value & Fdr & Enrichment score \\
\hline GO:0019221 & Cytokine-mediated signaling pathway & Biological process & 6 & 3.15545E-05 & 0.16723586 & 4.500939235 \\
\hline GO:0045071 & Negative regulation of viral genome replication & Biological process & 3 & $8.13812 E-05$ & 0.16723586 & 4.089475756 \\
\hline GO:0051707 & Response to other organism & Biological process & 8 & 0.000110304 & 0.16723586 & 3.957409854 \\
\hline GO:0043207 & Response to external biotic stimulus & Biological process & 8 & 0.000113542 & 0.16723586 & 3.944843243 \\
\hline GO:0009607 & Response to biotic stimulus & Biological process & 8 & 0.000146422 & 0.16723586 & 3.834392194 \\
\hline GO:0006955 & Immune response & Biological process & 9 & 0.000147975 & 0.16723586 & 3.829813087 \\
\hline GO:1903901 & Negative regulation of viral life cycle & Biological process & 3 & 0.000272286 & 0.263767544 & 3.564974349 \\
\hline GO:0045069 & Regulation of viral genome replication & Biological process & 3 & 0.000350815 & 0.297359886 & 3.454921375 \\
\hline GO:0048525 & Negative regulation of viral process & Biological process & 3 & 0.000516858 & 0.389424083 & 3.286628429 \\
\hline GO:0019079 & Viral genome replication & Biological process & 3 & 0.000615908 & 0.39115453 & 3.210484097 \\
\hline GO:0000782 & Telomere cap complex & Cellular component & 1 & 0.019429856 & 1 & 1.711530414 \\
\hline GO:0000783 & Nuclear telomere cap complex & Cellular component & 1 & 0.019429856 & 1 & 1.711530414 \\
\hline GO:0005883 & Neurofilament & Cellular component & 1 & 0.021177942 & 1 & 1.674116247 \\
\hline GO:0005576 & Extracellular region & Cellular component & 8 & 0.021620359 & 1 & 1.665137091 \\
\hline GO:0034709 & Methylosome & Cellular component & 1 & 0.022923006 & 1 & 1.63972844 \\
\hline GO:0005687 & U4 snRNP & Cellular component & 1 & 0.024665052 & 1 & 1.607917962 \\
\hline GO:0034719 & SMN-Sm protein complex & Cellular component & 1 & 0.031603169 & 1 & 1.500269361 \\
\hline GO:0071004 & U2-type prespliceosome & Cellular component & 1 & 0.031603169 & 1 & 1.500269361 \\
\hline GO:0005615 & Extracellular space & Cellular component & 6 & 0.035593759 & 1 & 1.448626141 \\
\hline GO:0098802 & Plasma membrane receptor complex & Cellular component & 2 & 0.039506187 & 1 & 1.40333488 \\
\hline GO:0005125 & Cytokine activity & Molecular function & 4 & 0.000123343 & 0.1554123 & 3.908885157 \\
\hline GO:0005126 & Cytokine receptor binding & Molecular function & 4 & 0.000804967 & 0.507128913 & 3.094222177 \\
\hline GO:0008009 & Chemokine activity & Molecular function & 2 & 0.001352491 & 0.568046338 & 2.868865526 \\
\hline GO:0098772 & Molecular function regulator & Molecular function & 8 & 0.002030662 & 0.639658597 & 2.692362313 \\
\hline GO:0042379 & Chemokine receptor binding & Molecular function & 2 & 0.003183034 & 0.733701618 & 2.497158668 \\
\hline GO:0048018 & Receptor ligand activity & Molecular function & 4 & 0.003493817 & 0.733701618 & 2.456699818 \\
\hline GO:0030545 & Receptor regulator activity & Molecular function & 4 & 0.004505466 & 0.810983968 & 2.346260236 \\
\hline GO:0004791 & Thioredoxin-disulfide reductase activity & Molecular function & 1 & 0.016139095 & 1 & 1.792120819 \\
\hline GO:0070990 & snRNP binding & Molecular function & 1 & 0.016139095 & 1 & 1.792120819 \\
\hline GO:0004312 & Fatty acid synthase activity & Molecular function & 1 & 0.017593822 & 1 & 1.754639806 \\
\hline
\end{tabular}

TABLE 5 | Detail information of IncRNAs and mRNAs with hyper- and hypo-methylation detected by $\mathrm{m}^{6} \mathrm{~A}$ single-base site qPCR.

\begin{tabular}{|c|c|c|c|c|c|}
\hline GeneSymbol & Transcript_ID & Position & Sequence context & Score & Decision \\
\hline LOC103693543 & XR_595034 & 1359 & 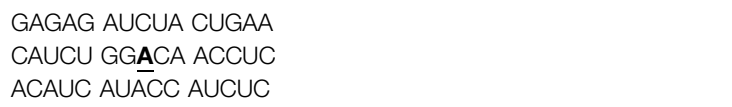 & 0.674 & $\begin{array}{l}\mathrm{m}^{6} \mathrm{~A} \text { site (very high } \\
\text { confidence) }\end{array}$ \\
\hline LOC102552786 & XR_346771 & 1712 & $\begin{array}{l}\text { GGUCC UCCAA UGAAU } \\
\text { UCAAA GGACA AUAGG } \\
\text { CGACC ACCAG UGAAU }\end{array}$ & 0.707 & $\begin{array}{l}\mathrm{m}^{6} \mathrm{~A} \text { site (very high } \\
\text { confidence) }\end{array}$ \\
\hline LOC102547952 & XR_338486 & 1546 & $\begin{array}{l}\text { CUGCA ACAUG GGAAG } \\
\text { AAGAG GGACA UAAGA } \\
\text { GAGGA CCCCA CCCCC }\end{array}$ & 0.676 & $\begin{array}{l}\mathrm{m}^{6} \mathrm{~A} \text { site (very high } \\
\text { confidence) }\end{array}$ \\
\hline LOC102548402 & XR_596592 & 3965 & $\begin{array}{l}\text { AUCUG ACGGC AGGAU } \\
\text { UUGGA GGACA CCUCU } \\
\text { GAAAG GGCCC CAGAA }\end{array}$ & 0.677 & $\begin{array}{l}\mathrm{m}^{6} \mathrm{~A} \text { site (very high } \\
\text { confidence) }\end{array}$ \\
\hline Clec1b & ENSRNOT00000082537 & 831 & $\begin{array}{l}\text { ACCCA GCTTC CTGTA CAGAG AGACA TTACT TAATA TGTGA } \\
\text { GAGAA }\end{array}$ & 0.731 & $\begin{array}{l}\mathrm{m}^{6} \mathrm{~A} \text { site (very high } \\
\text { confidence) }\end{array}$ \\
\hline Tnfrsf26 & ENSRNOT00000066943 & 416 & $\begin{array}{l}\text { CAGGA ATGCA ATGCC ACAAT GGAECA CTGTG TGTGA CTCCA } \\
\text { AGCAA }\end{array}$ & 0.634 & $\mathrm{~m}^{6} \mathrm{~A}$ site (high confidence) \\
\hline Ptk2b & ENSRNOT00000030007 & 3278 & $\begin{array}{l}\text { GTGCT ACTTG GGCTA CATCT GGACA GAAAG GACTC TGGGC } \\
\text { ACAGA }\end{array}$ & 0.732 & $\begin{array}{l}\mathrm{m}^{6} \mathrm{~A} \text { site (very high } \\
\text { confidence) }\end{array}$ \\
\hline Tnfrsf21 & ENSRNOT00000015918 & 928 & $\begin{array}{l}\text { CTGGG GTGTG AGGAA GAAAG GGA_A GAGAA TGAAG ATGTG } \\
\text { CGGTG }\end{array}$ & 0.747 & $\begin{array}{l}\mathrm{m}^{6} \mathrm{~A} \text { site (very high } \\
\text { confidence) }\end{array}$ \\
\hline Stk38l & NM_001083336 & 2090 & $\begin{array}{l}\text { ACCGG CTGCA AGGAA CTTAA GGACA CTGACTCCGA CATTA } \\
\text { GAATT }\end{array}$ & 0.640 & $\mathrm{~m}^{6} \mathrm{~A}$ site (high confidence) \\
\hline Ankrd54 & ENSRNOT00000014413 & 1173 & $\begin{array}{l}\text { CCCTC TGGCT GTTAG GGAAG GGACA GGAAC CCCAG AACAG } \\
\text { AGGAA }\end{array}$ & 0.643 & $\mathrm{~m}^{6} \mathrm{~A}$ site (high confidence) \\
\hline
\end{tabular}




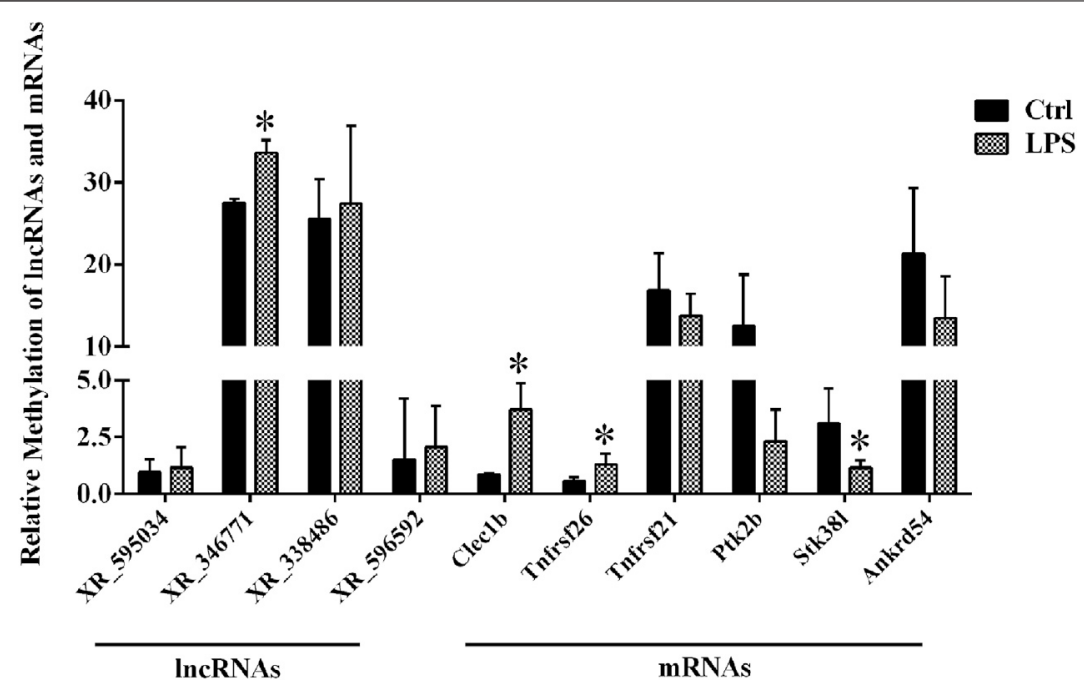

FIGURE 4 | M $\mathrm{M}^{6} \mathrm{~A}$ single-base site qPCR was used to confirm the microarray data for the top four methylated IncRNAs and top six methylated mRNAs in left ventricle tissue between the LPS and control groups. * $p<0.05$ versus the Ctrl group. Ctrl, control; LPS, lipopolysaccharide.

difference (Figure 1C). In addition, we measured the protein expression of METTL3/14, WTAP, and YTHDF1/3. We found that protein expressions of these proteins significantly downregulated in LPS group (Supplementary Figure S1). The expressions of METTL3/14, WTAP, and YTHDF1/3 were decreased by $42.2,64,47.6,49.6$ and $56.6 \%$, respectively. The results of protein expression and mRNA expression are consistent.

\section{$M^{6} A$ Modification Profiles of IncRNAs and mRNAs}

Probes specific for 27,770 mRNAs and 10,582 lncRNAs were used to analyze the samples from the LPS and control groups using an $\mathrm{m}^{6} \mathrm{~A}$ mRNA and lncRNA Arraystar epitranscriptomic microarray. The results showed that the $\mathrm{m}^{6} \mathrm{~A}$ modification levels of 62 mRNAs and 11 lncRNAs were significantly altered in the LPS group compared with control group (fold change $>1.5$, $p$-value $<0.05$ ) (Figures 2A,B). The altered lncRNAs and mRNAs were aligned for cluster analysis (Figures 2C,D). Based on their fold changes, we selected 11 lncRNAs with significantly altered $\mathrm{m}^{6} \mathrm{~A}$ modification levels and the top 10 up- and downregulated mRNAs to present the results (Tables 1, 2).

The data that support the findings of this study are openly available in the GenBank databases under accession number GSE159309.

\section{GO and KEGG Analyses of Differentially Methylated mRNAs}

$\mathrm{GO}$ analysis of the mRNAs with increased and decreased $\mathrm{m}^{6} \mathrm{~A}$ modification levels was performed. Among the mRNAs with decreased $\mathrm{m}^{6} \mathrm{~A}$ modification levels, the "cytokine-mediated signaling pathway" in BP, "immune response" in BP and "cytokine activity" in MF were revealed to have the maximum enrichment scores for GO terms (Figures 3A,B; Tables 3, 4).

KEGG analysis of the mRNAs with increased and decreased $\mathrm{m}^{6} \mathrm{~A}$ methylation modification levels were also conducted. The results showed that the mRNAs with increased $\mathrm{m}^{6} \mathrm{~A}$ modification levels were enriched in only one pathway, namely, the "PPAR signaling pathway" (Figure 3C). The mRNAs with decreased $\mathrm{m}^{6} \mathrm{~A}$ modification levels were enriched in 27 pathways, and the following main pathways reached higher enrichment scores: "IL17 signaling pathway", "TNF signaling pathway" and "Cytokinecytokine receptor interaction” (Figure 3D).

\section{qPCR Results of a Single-Base $\mathbf{m}^{6} \mathrm{~A}$ Site}

By using the SRAMP database, we predicted the mRNAs and lncRNAs with differential $\mathrm{m}^{6} \mathrm{~A}$ modification in the order from high to low fold change of $\mathrm{m}^{6} \mathrm{~A}$ modification. As a result, we identified four lncRNAs and six mRNAs with $\mathrm{m}^{6} \mathrm{~A}$ ACA sites and with high confidence scores of $\mathrm{qPCR}$ validation of single-base $\mathrm{m}^{6} \mathrm{~A}$ sites. The Detail information of $\operatorname{lncRNAs}$ and mRNAs detected by $\mathrm{m}^{6} \mathrm{~A}$ single-base site $\mathrm{qPCR}$ was shown in Table 5 . The results showed that among the $\operatorname{lncRNAs}, \mathrm{m}^{6} \mathrm{~A}$ modification levels were significantly increased in XR_346771. Among the mRNAs, $m^{6} \mathrm{~A}$ modification levels were significantly increased in C-type lectin domain family 1 member B (Clec1b) and tumor necrosis factor receptor superfamily member 26 (Tnfrsf26) and significantly decreased in serine/threonine kinase 38 like (STK38L) (Figure 4).

\section{PPI Analysis of Targeted Genes}

By employing the STRING database, we performed PPI analysis of Clec1b, Stk38l, and Tnfrsf26 (Figures 5A-C). KEGG analysis revealed that the $\mathrm{Clec} 1 \mathrm{~b}$ interaction-related proteins were mainly enriched in "platelet activation" and other pathways. The Stk381 

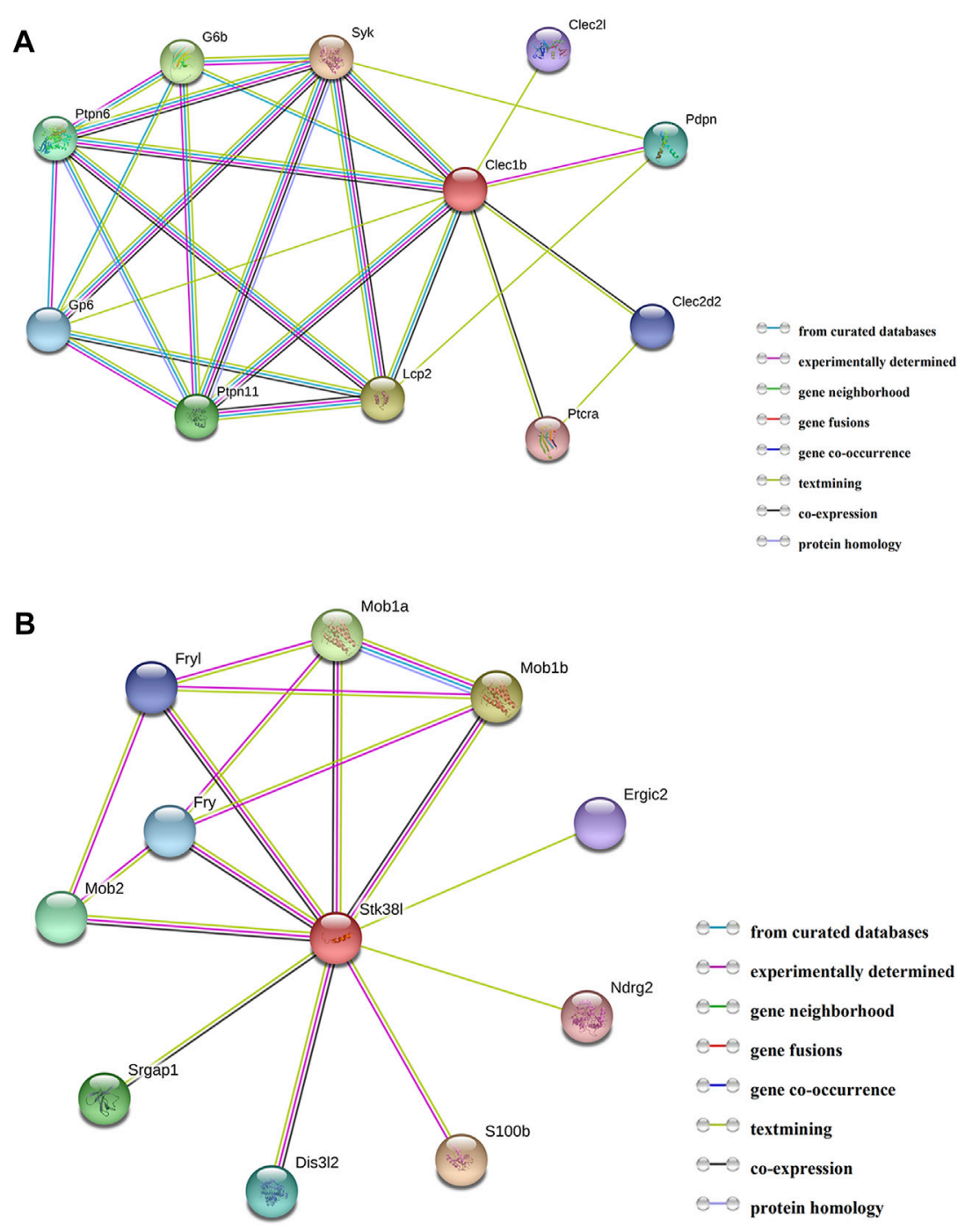

$$
\begin{aligned}
& \odot-\odot \text { from curated databases } \\
& \odot-\odot \text { experimentally determined } \\
& \odot-\odot \text { gene neighborhood } \\
& \odot-\odot \text { gene fusions } \\
& \odot-\odot \text { gene co-occurrence } \\
& \odot-\odot \text { textmining } \\
& \odot-\odot \text { co-expression } \\
& \odot-\odot \text { protein homology }
\end{aligned}
$$

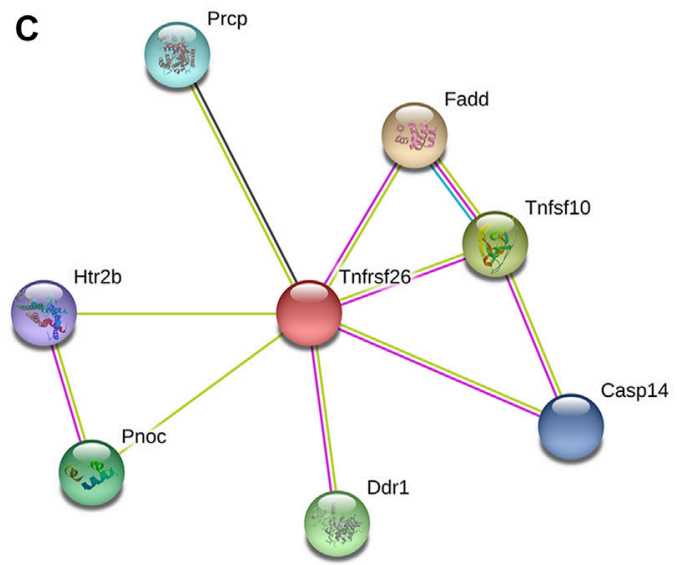

$$
\begin{aligned}
& \odot-\odot \text { from curated databases } \\
& \odot-\odot \text { experimentally determined } \\
& \odot-\odot \text { gene neighborhood } \\
& \odot-\odot \text { gene fusions } \\
& \odot-\odot \text { gene co-occurrence } \\
& \odot-\odot \text { textmining } \\
& \odot-\odot \text { co-expression } \\
& \odot-\odot \text { protein homology }
\end{aligned}
$$

FIGURE 5|Protein-protein interaction analysis. (A) PPI network of Clec1b, (B) PPI network of Stk38I, (C) PPI network of Tnfrsf26. PPI, protein-protein interaction; Clec1b, C-type lectin domain family 1 member B; Tnfrsf26, tumor necrosis factor receptor superfamily member 26; STK38L, serine/threonine kinase 38 like. 
TABLE 6 | Pathways of the matching proteins in the PPI network.

\begin{tabular}{|c|c|c|c|c|}
\hline Target gene & Term ID & Term description & FDR & Matching proteins in the network \\
\hline \multirow[t]{9}{*}{ Clec1b } & rno04650 & Natural killer cell mediated cytotoxicity & 0.000002500 & Lcp2, Ptpn11, Ptpn6, Syk \\
\hline & rno04611 & Platelet activation & $0.000360,000$ & Gp6, Lcp2, Syk \\
\hline & rno04662 & B Cell receptor signaling pathway & 0.004900000 & Ptpn6, Syk \\
\hline & rno04664 & Fc epsilon RI signaling pathway & 0.004900000 & Lcp2, Syk \\
\hline & rno04660 & T Cell receptor signaling pathway & 0.006500000 & Lcp2, Ptpn6 \\
\hline & rno04380 & Osteoclast differentiation & 0.007300000 & Lcp2, Syk \\
\hline & rno04072 & Phospholipase D signaling pathway & 0.009700000 & Ptpn11, Syk \\
\hline & rno04630 & Jak-STAT signaling pathway & 0.009700000 & Ptpn11, Ptpn6 \\
\hline & rno05205 & Proteoglycans in cancer & 0.012500000 & Ptpn11, Ptpn6 \\
\hline \multirow[t]{2}{*}{ Stk38| } & rno04392 & Hippo signaling pathway - multiple species & 0.000260000 & Mob1a, Mob1b \\
\hline & rno04390 & Hippo signaling pathway & 0.003500000 & Mob1a, Mob1b \\
\hline \multirow[t]{2}{*}{ Tnfrsf26 } & rno04210 & Apoptosis & 0.023800000 & Fadd, Tnfsf10 \\
\hline & rno04217 & Necroptosis & 0.023800000 & Fadd, Tnfsf10 \\
\hline
\end{tabular}

interaction-related proteins were mainly enriched in the "hippo signaling pathway", and the Tnfrsf 26 interaction-related proteins were mainly enriched in "apoptosis" (Table 6).

\section{ceRNA Analysis of IncRNA XR_346771}

A ceRNA network of the lncRNA-microRNA-mRNA interaction network was constructed for XR_346,771 (Figure 6A). GO and KEGG analyses were performed on the predicted mRNAs (Figures 6B,C). The "inorganic cation import across plasma membrane", "transmembrane transport", and "import across plasma membrane" in BP were significantly enriched for GO terms (Figure 6B). FAM155B and PICALM are downstream proteins related to transmembrane transport that are regulated by XR_346771, and the corresponding sponged miRNAs are shown in Table 7.

\section{DISCUSSION}

Our study suggested that the $\mathrm{m}^{6} \mathrm{~A}$ modification level was decreased in septic heart tissues. Our genome-wide screening of $\mathrm{m}^{6} \mathrm{~A}$-tagged transcript profiles indicated that the $\mathrm{m}^{6} \mathrm{~A}$ modification levels in $39 \mathrm{mRNAs}$ and 7 lncRNAs were significantly decreased and that the $\mathrm{m}^{6} \mathrm{~A}$ modification levels in 23 mRNAs and four lncRNAs were significantly increased.

Pathway analyses of mRNAs with altered m6A modification were conducted in our study. The results showed that the mRNAs with decreased $\mathrm{m}^{6} \mathrm{~A}$ modification levels were enriched in many pathways related to immune and inflammatory responses. In recent years, the roles of $\mathrm{m}^{6} \mathrm{~A}$ modification in autoimmune and inflammatory diseases have attracted substantial attention (Rubio et al., 2018). METTL3 is a key enzyme of $\mathrm{m}^{6} \mathrm{~A}$ methylation modification and is involved in immune and inflammatory regulation. Feng et al. found that METTL3 inhibits inflammation by affecting the alternative splicing of MyD88 (Feng et al., 2018). METTL3 expression is significantly upregulated in blood samples from patients with rheumatoid arthritis. In addition, METTL3 attenuates LPS-induced macrophage-mediated inflammation through the NF- $\kappa \mathrm{B}$ pathway (Wang et al., 2019a). The $\mathrm{m}^{6} \mathrm{~A}$ "reader" YT521-B homology domain family 2 (YTHDF2) inhibits HCC cells and tumor vasculature by degrading the mRNAs of IL11 and serpin family E member 2 (Wang et al., 2019b). These findings indicate the important role of $\mathrm{m}^{6} \mathrm{~A}$ modification in inflammatory and immune disorders. Sepsis is mainly caused by the host's unbalanced response to infection. In sepsis, microbial infections or necrotic tissues release a large amount of harmful substances, leading to the activation of the systemic immune response and the excessive activation of immune cells. The excessive release of cytokines is destructive. There is currently no effective treatment for sepsis because in the past few decades, attempts to use anti-inflammatory treatments to limit the tissue damage caused by excessive inflammation have failed (Zeni et al., 1997). Our study suggests that alterations in $\mathrm{m}^{6} \mathrm{~A}$ modification in sepsis are closely related to inflammatory and immune responses. Since we study cardiac tissue, we speculate that $\mathrm{m}^{6} \mathrm{~A}$ modification plays an important role in sepsis-induced cardiac function dysfunction.

We selected four lncRNAs and six mRNAs for the qPCR validation of single-base $\mathrm{m}^{6} \mathrm{~A}$ sites in our study. The results showed that among the lncRNAs, the $\mathrm{m}^{6} \mathrm{~A}$ modification levels were significantly increased in XR_346771. Among the mRNAs, the $\mathrm{m}^{6} \mathrm{~A}$ modification levels were significantly increased in Clec1b and Tnfrsf26 and significantly decreased in Stk38l. These results are consistent with those of high-throughput sequencing; therefore, we attempted to conduct an in-depth analysis of this lncRNA and the three mRNAs.

First, we performed PPI analysis of Clec1b, STK38L, and Tnfrsf26 to investigate the downstream possible regulatory genes and biological functions of these proteins. The Cleclb-interacting proteins are enriched in the pathway of "platelet activation". C-type lectin-like receptor 2 (CLEC-2) is a protein encoded by the Clec1b gene. Platelets play a critical role in innate and adaptive immunity (Semple et al., 2011). Platelets can alleviate LPS-induced septic shock by regulating the ability of macrophages to engulf and kill bacteria (Xiang et al., 2013). In two mouse models of sepsis (intraperitoneal LPS injection and cecal ligation and puncture), platelet CLEC-2 reduced the severity of sepsis by controlling the migration of monocytes/macrophages to the infection site, the expression of inflammatory mediators, and damage to organs (Rayes et al., 2017). In our sepsis model, we found that the $\mathrm{m}^{6} \mathrm{~A}$ modification level of Cleclb mRNA was significantly increased. 


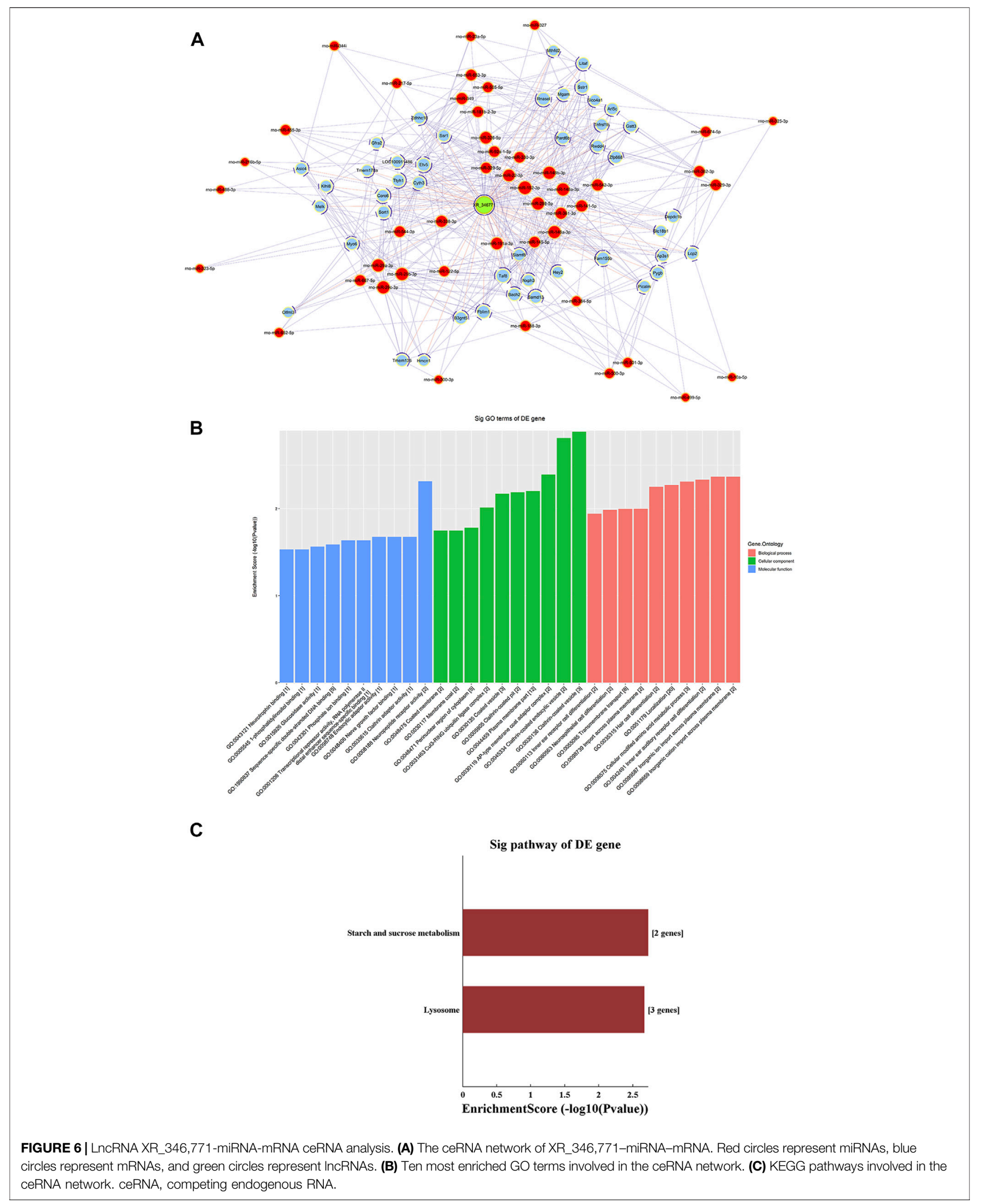


TABLE 7 | LncRNA XR_346771targeted miRNAs and mRNAs.

LncRNA

XR_346771
Targeted miRNA

Targeted mRNA

PICALM

FAM155B miR-148b-3p, miR-152-3p, miR-361-3p, miR-384-5p, miR-500-3p, miR-501-3p miR-122-5p, miR-146a-3p, miR-188-3p, miR-298-5p, miR-326-5p, miR-329-5p, miR-361-3p, miR-500-3p, miR-501-3p, miR-542-3p, miR-674-5p, miR-92a-1-5p

Considering that the degree and pattern of $\mathrm{m}^{6} \mathrm{~A}$ modification may affect the transport, splicing, storage, translation, stability, and decay of mRNAs, we speculated that the elevated level of Clec1b $\mathrm{m}^{6} \mathrm{~A}$ modification affects the functions of Cleclb and associate with activation of platelets. The interacting proteins of Cleclb were glycoprotein VI (Gp6), lymphocyte cytosolic protein 2 (Lcp2), and spleen tyrosine kinase (Syk). These proteins might be used as target proteins for in-depth research.

Our study suggests that the $\mathrm{m}^{6} \mathrm{~A}$ modification level of Tnfrsf 26 mRNA is increased and of Stk381 mRNA is decreased. According to PPI network analysis, Stk38l-interacting proteins are enriched in the "Hippo signaling pathway" pathway. Hippo signaling pathway may promote cell differentiation and death and inhibit cell proliferation (Chang et al., 2019). Tnfrsf26-interacting proteins are mainly enriched in the pathway of apoptosis. Sepsis-induced cardiac dysfunction has been reported by many studies to be closely related to apoptosis. When LPS stimulates H9C2 cells (rat embryonic cardiomyoblasts), the overexpression of miR-146a suppresses apoptosis and helps attenuate myocardial depression (An et al., 2018). Chao et al. found that the overexpression of Tid1$\mathrm{S}$ can enhance ER- $\alpha$ to activate p-PI3K/p-Akt, attenuating LPSinduced apoptosis in H9C2 cells (Chao et al., 2019). In recent years, as the understanding of $\mathrm{m}^{6} \mathrm{~A}$ modification has gradually deepened, studies have found that $\mathrm{m}^{6} \mathrm{~A}$ modification plays an important role in the process of apoptosis. In the human glioma cell line U251, lower level of $\mathrm{m}^{6} \mathrm{~A}$ modification can reduce apoptosis and promote cell proliferation (Li et al., 2019). Moreover, METTL3 can regulate the expression of several important proteins that regulate the survival, apoptosis, invasion, and proliferation of lung cancer cells (Lin et al., 2016). We hypothesized that the $\mathrm{m}^{6} \mathrm{~A}$ modification of Tnfrsf26 and Stk38l might be related to cardiomyocyte apoptosis.

LncRNAs are a class of noncoding RNAs that are more than 200 nucleotides in length. In recent years, $\mathrm{m}^{6} \mathrm{~A}$-modified $\mathrm{lncRNAs}$ have received extensive attention. $\mathrm{M}^{6} \mathrm{~A}$ modification may control gene expression by regulating the translation efficiency and stability of lncRNAs (Coker et al., 2019). For example, the $\mathrm{m}^{6} \mathrm{~A}$-modified lncRNA MALAT1 is implicated in ischemia reperfusion injuryinduced inflammation (Yang et al., 2020). LncRNAs contain miRNA-responsive elements, which function as ceRNAs, interact with miRNAs and indirectly regulate mRNAs. In the present study, we established and analyzed a potential ceRNA network of XR_346771 through bioinformatics analysis. We performed GO and KEGG analyses of the predicted mRNAs to further explore the function of XR_346771.

GO analysis showed that "inorganic cation import across plasma membrane", "inorganic ion import across plasma membrane", "transmembrane transport", and "import across plasma membrane" were significantly enriched. Cardiac contraction requires $\mathrm{Ca}^{2+}$, so abnormal $\mathrm{Ca}^{2+}$ homeostasis may play a key role in the pathogenesis of common cardiovascular diseases. Studies have reported that $\mathrm{m}^{6} \mathrm{~A}$ modification promotes neurite extension and neuronal differentiation by acting as a $\mathrm{Ca}^{2+}$ channel (Mukobata et al., 2002). In 6-OHDA-induced PC12 cells and the cerebral striatum of rats with Parkinson's disease, decreased $\mathrm{m}^{6} \mathrm{~A}$ modification induces the expression of $\mathrm{N}$-methyl-D-aspartate receptor 1 and increases oxidative stress and $\mathrm{Ca}^{2+}$ influx, leading to apoptosis of dopaminergic neurons (Chen et al., 2019). These studies indicate that $\mathrm{m}^{6} \mathrm{~A}$ modification is involved in $\mathrm{Ca}^{2+}$ transport. We speculated that the $\mathrm{m}^{6} \mathrm{~A}$ modification of XR_346771 might associate with abnormal $\mathrm{Ca}^{2+}$ homeostasis in cardiac tissues.

It is interesting that even though the methyltransferase complex was downregulated upon sepsis induced myocardial dysfunction; there were still 27 transcripts that were significantly hypermethylated. Chokkalla et al. found that, compared with sham group, global $\mathrm{m}^{6} \mathrm{~A}$ increased significantly at 12 and $24 \mathrm{~h}$ of reperfusion in transient middle cerebral artery occlusion in C57BL/6J mice. The FTO decreased significantly after stroke compared with sham. While 139 transcripts (122 mRNAs and 17 lncRNAs) were hypermethylated, 8 transcripts (5 mRNAs and 3 lncRNAs) were hypomethylated in the ischemic brain at $12 \mathrm{~h}$ reperfusion (Chokkalla et al., 2019). Wang et al. found that METTL3 was downregulated after traumatic brain injury in mice. In total, $922 \mathrm{~m}^{6} \mathrm{~A}$ peaks were differentially expressed as determined by $\mathrm{m}^{6} \mathrm{~A}-\mathrm{RIP}$-seq, with 370 upregulated and 552 downregulated methylated mRNA (Wang et al., 2019b). These studies are consistent with our research. Previous research indicated that the methylation modification of $\mathrm{m}^{6} \mathrm{~A}$ has been proved to be reversible, as it modulated by RNA methyltransferases, RNA-binding proteins and demethylases. The $\mathrm{m}^{6} \mathrm{~A}$ methyltransferase, also known as "Writers", including METTL3/14, WTAP, KIAA1429 and RNA binding motifs protein 15/15B (RBM15/15B), E3 ubiquitin-protein ligase Hakai (HAKAI) and zinc finger CCCH-type containing 13 (ZC3H13). The RNA-binding proteins, also known as "readers", including: YTHDF1/2/3, YTH domain-containing reader proteins $1 / 2$ (YTHDC1/2), insulin-like growth factor 2 mRNA-binding proteins $1 / 2 / 3$ (IGF2BP1/2/3), epitheliumspecific ETS (ESE) transcription factors (ELF3), heterogeneous nuclear ribonucleoprotein A2B1 (hnRNPA2B1). The $\mathrm{m}^{6} \mathrm{~A}$ demethylase, also known as "eraser", including: FTO, a-ketoglutarate-dependent dioxygenase alkB homologue 5 (ALKHB5). In our research we tested mRNA level of some enzymes, but not all enzymes. Therefore, there may be other enzymes involved in $\mathrm{m}^{6} \mathrm{~A}$ modification upon sepsis induced 
myocardial dysfunction. The $\mathrm{m}^{6} \mathrm{~A}$ modification status of specific RNA was catalyzed by different enzymes. METTL3 positively regulates expression of MYD88, a critical upstream regulator of NF- $\kappa B$ signaling, by facilitating $\mathrm{m}^{6} \mathrm{~A}$ methylation modification to MYD88-RNA in mesenchymal stem cells (Yu et al., 2020). However, reduced expression of FTO increased Nanog mRNA $\mathrm{m}^{6} \mathrm{~A}$ methylation under TNF- $\alpha$ stimulation, decreased Nanog mRNA and protein expression, and significantly inhibited mesenchymal stem cells capacity for differentiation to sweat gland cells (Wang et al., 2020). In our research, these transcripts that have undergone $\mathrm{m}^{6} \mathrm{~A}$ modification might have been modified by different enzymes.

In conclusion, our experiments revealed that the level of $\mathrm{m}^{6} \mathrm{~A}$ modification is significantly decreased in septic cardiac tissue. Through the genome-wide profiling of $\mathrm{m}^{6} \mathrm{~A}$-tagged mRNAs and lncRNAs and subsequent bioinformatics analysis, we revealed some potential functions of transcripts with altered $\mathrm{m}^{6} \mathrm{~A}$ modification.

\section{DATA AVAILABILITY STATEMENT}

The datasets presented in this study can be found in online repositories. The names of the repository/repositories and accession number(s) can be found below: https://www.ncbi. nlm.nih.gov/genbank/, GSE159309.

\section{ETHICS STATEMENT}

The animal study was reviewed and approved by The Experimental Animal Welfare Ethics Branch and the Biomedical Ethics Committee of Peking University (LA 2020343).

\section{REFERENCES}

An, R., Feng, J., Xi, C., Xu, J., and Sun, L. (2018). miR-146a Attenuates SepsisInduced Myocardial Dysfunction by Suppressing IRAK1 and TRAF6 via Targeting ErbB4 Expression. Oxid. Med. Cell Longev. 2018, 1-9. doi:10.1155/2018/7163057

Arcidiacono, O. A., Krejčí, J., and Bártová, E. (2020). The Distinct Function and Localization of METTL3/METTL14 and METTL16 Enzymes in Cardiomyocytes. Int. J. Mol. Sci. 21, 8139. doi:10.3390/ijms21218139

Berulava, T., Buchholz, E., Elerdashvili, V., Pena, T., Islam, M. R., Lbik, D., et al. (2020). Changes in m6A RNA Methylation Contribute to Heart Failure Progression by Modulating Translation. Eur. J. Heart Fail. 22, 54-66. doi:10.1002/ejhf.1672

Chang, Y., Fu, X. R., Cui, M., Li, W. M., Zhang, L., Li, X., et al. (2019). Activated Hippo Signal Pathway Inhibits Cell Proliferation and Promotes Apoptosis in NK/T Cell Lymphoma Cells. Cancer Med. 8, 3892-3904. doi:10.1002/ cam 4.2174

Chao, C. N., Lo, J. F., Khan, F. B., Day, C. H., Lai, C. H., Chen, C. H., et al. (2019). Tid1-S Attenuates LPS-induced Cardiac Hypertrophy and Apoptosis through ER-a Mediated Modulation of p-PI3K/p-Akt Signaling cascade. J. Cel Biochem. 120, 16703-16710. doi:10.1002/jcb.28928

Chen, X., Yu, C., Guo, M., Zheng, X., Ali, S., Huang, H., et al. (2019). DownRegulation of m6A mRNA Methylation Is Involved in Dopaminergic Neuronal Death. ACS Chem. Neurosci. 10, 2355-2363. doi:10.1021/ acschemneuro.8b00657

Chokkalla, A. K., Mehta, S. L., Kim, T., Chelluboina, B., Kim, J., and Vemuganti, R. (2019). Transient Focal Ischemia Significantly Alters the M 6 A

\section{AUTHOR CONTRIBUTIONS}

$\mathrm{S}-\mathrm{YZ}$ and $\mathrm{Y}-\mathrm{CH}$ conceived and planned the experiments. $\mathrm{Y}-\mathrm{CH}$, $\mathrm{H}-\mathrm{ZX}$, and BL performed the experiment and acquired the data. R-LX, H-PZ, and J-YL analyzed and interpreted the data. Y-CH drafted the manuscript. S-YZ revised the manuscript. All authors provided critical feedback and help in shaping the research, analysis, and manuscript. All authors have read and approved the final submitted manuscript.

\section{FUNDING}

This work was supported by the Chinese Academy of Medical Sciences (CAMS) Innovation Fund for Medical Sciences (No. 2016-I2M-1-011). The funders had no role in study design, data collection and analysis, decision to publish, or preparation of the manuscript.

\section{SUPPLEMENTARY MATERIAL}

The Supplementary Material for this article can be found online at: https://www.frontiersin.org/articles/10.3389/fmolb.2021.670160/ full\#supplementary-material

Supplementary Figure $\mathbf{1} \mid$ Expressions of $\mathrm{m}^{6} \mathrm{~A}$-related enzymes were detected by western blot analysis. $n=3-4$; ${ }^{\star} P<0.05$. Ctrl, control; LPS, lipopolysaccharide.

Supplementary Table 1 | Sequence of primers used for qRT-PCR analysis of mRNA levels.

Supplementary Table 2 | Sequence of primers used for $m^{6} A$ single base site qPCR analysis of relative mRNA methylation levels.

Epitranscriptomic Tagging of RNAs in the Brain. Stroke 50, 2912-2921. doi:10.1161/strokeaha.119.026433

Cohen, J., Vincent, J.-L., Adhikari, N. K. J., Machado, F. R., Angus, D. C., Calandra, T., et al. (2015). Sepsis: a Roadmap for Future Research. Lancet Infect. Dis. 15, 581-614. doi:10.1016/s1473-3099(15)70112-x

Coker, H., Wei, G., and Brockdorff, N. (2019). m6A Modification of Non-coding RNA and the Control of Mammalian Gene Expression. Biochim. Biophys. Acta Gene Regul. Mech. 1862, 310-318. doi:10.1016/j.bbagrm.2018.12.002

Enright, A. J., John, B., Gaul, U., Tuschl, T., Sander, C., and Marks, D. S. (2003). MicroRNA Targets in Drosophila. Genome Biol. 5, R1. doi:10.1186/gb-2003-5-1-r1

Feng, Z., Li, Q., Meng, R., Yi, B., and Xu, Q. (2018). METTL 3 Regulates Alternative Splicing of MyD88 upon the Lipopolysaccharide-induced Inflammatory Response in Human Dental Pulp Cells. J. Cel Mol. Med. 22, 2558-2568. doi:10.1111/jcmm.13491

Huang, T., Guo, J., Lv, Y., Zheng, Y., Feng, T., Gao, Q., et al. (2019). Meclofenamic Acid Represses Spermatogonial Proliferation through Modulating m6A RNA Modification. J. Anim. Sci. Biotechnol. 10, 63. doi:10.1186/s40104-019-0361-6

Imanishi, M., Tsuji, S., Suda, A., and Futaki, S. (2017). Detection ofN6Methyladenosine Based on the Methyl-Sensitivity of MazF RNA Endonuclease. Chem. Commun. 53, 12930-12933. doi:10.1039/c7cc07699a

Kennedy, E. M., Bogerd, H. P., Kornepati, A. V. R., Kang, D., Ghoshal, D., Marshall, J. B., et al. (2016). Posttranscriptional M 6 A Editing of HIV-1 mRNAs Enhances Viral Gene Expression. Cell Host Microbe 19, 675-685. doi:10.1016/j.chom.2016.04.002

Kmietczyk, V., Riechert, E., Kalinski, L., Boileau, E., Malovrh, E., Malone, B., et al. (2019). m6A-mRNA Methylation Regulates Cardiac Gene Expression and Cellular Growth. Life Sci. Alliance 2, e201800233. doi:10.26508/lsa.201800233 
Li, F., Zhang, C., and Zhang, G. (2019). m6A RNA Methylation Controls Proliferation of Human Glioma Cells by Influencing Cell Apoptosis. Cytogenet. Genome Res. 159, 119-125. doi:10.1159/000499062

Lin, S., Choe, J., Du, P., Triboulet, R., and Gregory, R. I. (2016). The M 6 A Methyltransferase METTL3 Promotes Translation in Human Cancer Cells. Mol. Cel 62, 335-345. doi:10.1016/j.molcel.2016.03.021

Lin, X., Chai, G., Wu, Y., Li, J., Chen, F., Liu, J., et al. (2019). RNA m6A Methylation Regulates the Epithelial Mesenchymal Transition of Cancer Cells and Translation of Snail. Nat. Commun. 10, 2065. doi:10.1038/s41467-019-09865-9

Mathiyalagan, P., Adamiak, M., Mayourian, J., Sassi, Y., Liang, Y., Agarwal, N., et al. (2019). FTO-dependent N 6 -Methyladenosine Regulates Cardiac Function during Remodeling and Repair. Circulation 139, 518-532. doi:10.1161/circulationaha.118.033794

Mukobata, S., Hibino, T., Sugiyama, A., Urano, Y., Inatomi, A., Kanai, Y., et al. (2002). M6a Acts as a Nerve Growth Factor-Gated Ca2+ Channel in Neuronal Differentiation. Biochem. Biophys. Res. Commun. 297, 722-728. doi:10.1016/s0006-291x(02)02284-2

Nie, M. W., Han, Y. C., Shen, Z. J., and Xie, H. Z. (2020). Identification of circRNA and mRNA Expression Profiles and Functional Networks of Vascular Tissue in Lipopolysaccharide-induced Sepsis. J. Cel Mol. Med. 24, 7915-7927. doi:10.1111/jcmm.15424

Pan, T. (2018). Modifications and Functional Genomics of Human Transfer RNA. Cell Res 28, 395-404. doi:10.1038/s41422-018-0013-y

Pasquinelli, A. E. (2012). MicroRNAs and Their Targets: Recognition, Regulation and an Emerging Reciprocal Relationship. Nat. Rev. Genet. 13, 271-282. doi:10.1038/nrg3162

Rayes, J., Lax, S., Wichaiyo, S., Watson, S. K., Di, Y., Lombard, S., et al. (2017). The Podoplanin-CLEC-2 axis Inhibits Inflammation in Sepsis. Nat. Commun. 8, 2239. doi:10.1038/s41467-017-02402-6

Rubio, R. M., Depledge, D. P., Bianco, C., Thompson, L., and Mohr, I. (2018). RNA m6A Modification Enzymes Shape Innate Responses to DNA by Regulating Interferon $\beta$. Genes Dev. 32, 1472-1484. doi:10.1101/gad.319475.118

Semple, J. W., Italiano, J. E., Jr., and Freedman, J. (2011). Platelets and the Immune Continuum. Nat. Rev. Immunol. 11, 264-274. doi:10.1038/nri2956

Song, H., Feng, X., Zhang, H., Luo, Y., Huang, J., Lin, M., et al. (2019). METTL3 and ALKBH5 Oppositely Regulate m6A Modification of TFEB mRNA, Which Dictates the Fate of Hypoxia/reoxygenation-Treated Cardiomyocytes. Autophagy 15, 1419-1437. doi:10.1080/15548627.2019.1586246

Wang, J., Yan, S., Lu, H., Wang, S., and Xu, D. (2019a). METTL3 Attenuates LPSInduced Inflammatory Response in Macrophages via NF- $\mathrm{kB}$ Signaling Pathway. Mediators Inflamm. 2019, 1-8. doi:10.1155/2019/3120391

Wang, Y., Mao, J., Wang, X., Lin, Y., Hou, G., Zhu, J., et al. (2019b). Genome-wide Screening of Altered m6A-Tagged Transcript Profiles in the hippocampus after Traumatic Brain Injury in Mice. Epigenomics 11, 805-819. doi:10.2217/epi-2019-0002

Wang, Y., Wang, R., Yao, B., Hu, T., Li, Z., Liu, Y., et al. (2020). TNF-a Suppresses Sweat Gland Differentiation of MSCs by Reducing FTO-Mediated m6A-Demethylation of Nanog mRNA. Sci. China Life Sci. 63, 80-91. doi:10.1007/s11427-019-9826-7
Xiang, B., Zhang, G., Guo, L., Li, X.-A., Morris, A. J., Daugherty, A., et al. (2013). Platelets Protect from Septic Shock by Inhibiting Macrophage-dependent Inflammation via the Cyclooxygenase 1 Signalling Pathway. Nat. Commun. 4, 2657. doi:10.1038/ncomms3657

Yang, C., Fan, Z., and Yang, J. (2020). m6A Modification of LncRNA MALAT1: A Novel Therapeutic Target for Myocardial Ischemia-Reperfusion Injury. Int. J. Cardiol. 306, 162. doi:10.1016/j.ijcard.2019.11.140

Yang, N., Shi, X.-L., Zhang, B.-L., Rong, J., Zhang, T.-N., Xu, W., et al. (2018). The Trend of $\beta 3$-Adrenergic Receptor in the Development of Septic Myocardial Depression: A Lipopolysaccharide-Induced Rat Septic Shock Model. Cardiology 139, 234-244. doi:10.1159/000487126

Yu, J., Shen, L., Liu, Y., Ming, H., Zhu, X., Chu, M., et al. (2020). The m6A Methyltransferase METTL3 Cooperates with Demethylase ALKBH5 to Regulate Osteogenic Differentiation through NF-kB Signaling. Mol. Cel Biochem. 463, 203-210. doi:10.1007/s11010-019-03641-5

Zaky, A., Deem, S., Bendjelid, K., and Treggiari, M. M. (2014). Characterization of Cardiac Dysfunction in Sepsis. Shock 41, 12-24. doi:10.1097/ shk.0000000000000065

Zeni, F., Freeman, B., and Natanson, C. (1997). Anti-inflammatory Therapies to Treat Sepsis and Septic Shock. Crit. Care Med. 25, 1095-1100. doi:10.1097/ 00003246-199707000-00001

Zhang, T.-N., Yang, N., Goodwin, J. E., Mahrer, K., Li, D., Xia, J., et al. (2019). Characterization of Circular RNA and microRNA Profiles in Septic Myocardial Depression: a Lipopolysaccharide-Induced Rat Septic Shock Model. Inflammation 42, 1990-2002. doi:10.1007/s10753-019-01060-8

Zhou, Y., Zeng, P., Li, Y.-H., Zhang, Z., and Cui, Q. (2016). SRAMP: Prediction of Mammalian N6-Methyladenosine (m6A) Sites Based on Sequence-Derived Features. Nucleic Acids Res. 44, e91. doi:10.1093/nar/gkw104

Conflict of Interest: The authors declare that the research was conducted in the absence of any commercial or financial relationships that could be construed as a potential conflict of interest.

Publisher's Note: All claims expressed in this article are solely those of the authors and do not necessarily represent those of their affiliated organizations, or those of the publisher, the editors and the reviewers. Any product that may be evaluated in this article, or claim that may be made by its manufacturer, is not guaranteed or endorsed by the publisher.

Copyright $\odot 2021$ Han, Xie, Lu, Xiang, Zhang, Li and Zhang. This is an open-access article distributed under the terms of the Creative Commons Attribution License (CC $B Y)$. The use, distribution or reproduction in other forums is permitted, provided the original author(s) and the copyright owner(s) are credited and that the original publication in this journal is cited, in accordance with accepted academic practice. No use, distribution or reproduction is permitted which does not comply with these terms. 